\title{
Computer-Guided Implant Surgery, an Overview from the Diagnosis to the Rehabilitation
}

\author{
Thiago de Almeida Prado Naves Carneiro DDS, MSc \& PhD*, Keuler Ferreira Rangel DDS, \\ Eder Ferreira Rangel DDS, Asbel Rodrigues Machado DDS, MSc \\ *Corresponding Author: Dr. Thiago de Almeida Prado Naves Carneiro, Federal University of \\ Uberlândia, Institute of Biomedical Sciences, School of Dentistry, Brazil, Email: tapncarneiro@hotmail \\ .com
}

\begin{abstract}
Treatment planning for the implanted prosthesis should begin long before the implant placement or even the choice of the implant itself. This is the concept of reverse planning. The appearance of computed tomography revolutionized imaging by obtaining three-dimensional anatomical structures with much more precision and definition. From the tomographic examination, it becomes possible to carry out a virtual surgical planning and generate surgical guides for implant placement. Because of this great conceptual revolution in implant treatment, the guided surgery emerges. With this technique, it is possible to perform implant placement surgery within the "flapless" concept, which means: surgery without incision or mucoperiosteal flap. For the application of this technique, it is essential that the guide remains perfectly adapted, with no possibility of changing position during the imaging examination and its fixation during the surgical procedure. In addition, it is possible to gain a lot of time in surgery and prosthetic steps, making the procedure much faster and more accurate. This article seeks to demystify the computer-guided surgery, showing its characteristics and discussing the technique from the beginning to the end, addressing possible failures and ways of avoiding them, seeking to extract the great advantages of this technique.
\end{abstract}

Keywords: Implant; Dental Implants; Guided Surgery; Virtual Planning; Flapless Surgery

\section{INTRODUCTION}

The discovery of osseointegration and the introduction of dental implants in the daily practice of dentistry revolutionized the oral rehabilitation. A subsequent revolution in implantology involves the use of threedimensional (3-D) planning software or computed programs to assist in the more efficient placement of implants in relation to anatomical and prosthetic needs(1). The development of 3-D surgical planning software combined with Computed Tomography images converted into 3-D models of the maxillofacial skeleton, allowed the visualization of the true representation of the patient's bone on the computer screen and, thus, performing a simulation of different treatment possibilities for that patient(2).

Originally, this concept of implant treatment planning was developed based on threedimensional guided surgery, in which the surgeon refers the patient to the radiologist, who examines the patient using a tomographic guide and a radiographic index. The radiographic guide is scanned separately after examination of the patient. The clinician converts the digital images (DICOM) files into a 3-D format of the patient's bone and prosthesis, aligned with radiopaque markers. The clinician plans the treatment of the patient in a virtual environment, orders a prototype surgical guide according to the treatment plan, which allows guiding the drills and implants during the surgery. This concept seemed to be the solution to the problems of all implant dentists. However, to achieve success with this technique, it is necessary to follow a series of clinical, radiological and laboratory steps so that accuracy can be achieved during the surgical procedure (3).

If otherwise, depending on the clinical situation, the results may be unpredictable. Due to possible failures in the execution stages and reliance on blind, flapless surgery, some unpredictable results began to emerge and the technique became questioned $(4,5,6)$.

Currently, there are different practical ways to perform this technique in a clinical environment, the most common of which are: guided surgery using stereolithography or prototyping drilled 
guides, laboratory-made guides or computer navigation systems. Computer navigation systems allow for a trans-operative analysis with real-time tracking. It is beyond the scope of this paper to evaluate or compare the accuracy of implant placement using prototype guides, laboratory guides, or computer guided surgical navigation. This article aims to demystify the guided surgery, showing its characteristics and discussing the technique from the beginning to the end, addressing possible failures and ways of avoiding them, seeking to extract the great advantages of this technique at the lowest risk possible.

\section{CASE REPORT}

A maxillary complete edentulous male patient, 54 years old, sought treatment with a primary complaint related to the instability of the upper complete denture he used (Fig. 1). After anamnesis and clinical examination, the treatment with implants and an upper fixed total hybrid prosthesis, also known as the Branemark protocol, were proposed. Initially, a new conventional maxillary complete denture was made, respecting the previously lost vertical dimension and all aesthetic / functional aspects (Fig. 2). After adapting the patient to this removable rehabilitation during a period of use of a few weeks, the complete denture was duplicated in a 1: 1 ratio of hyperdense material (Zinc Oxide + Acrylic Resin) (Fig. 3). The radiopaque replica of the prosthesis received a tomographic plate (Pross Guide/KEA Tech DabiAtlante - RibeirãoPreto, SP, Brazil) that was attached to its occlusal surface. A rigid occlusal registration with red acrylic resin was performed on the plate (Fig. 4). In this way, the tomographic guide is ready for the CT scan. The rigid bite registration allows reproducibility of the same position during tomographic examination and during surgery, minimizing the possibility of errors.

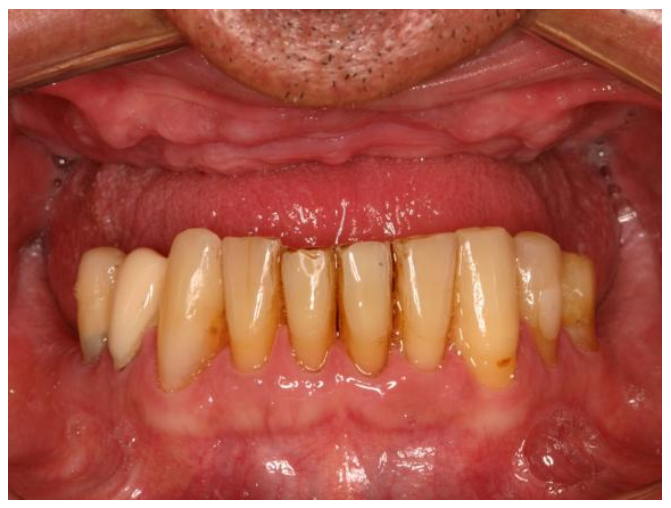

Fig1. Initial aspect

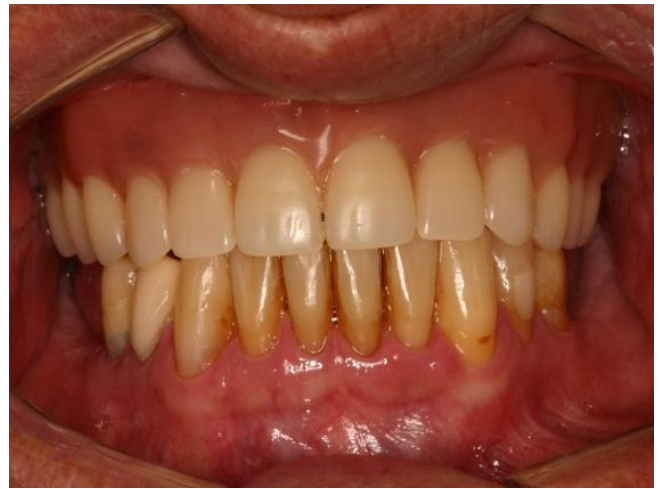

Fig2. Brand new maxillary complete denture

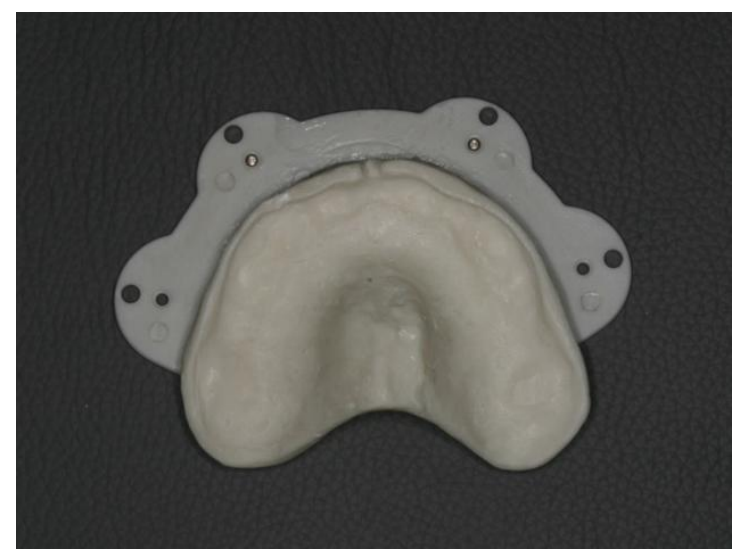

Fig3. The radiopaque replica of the prosthesis with the tomographic plate

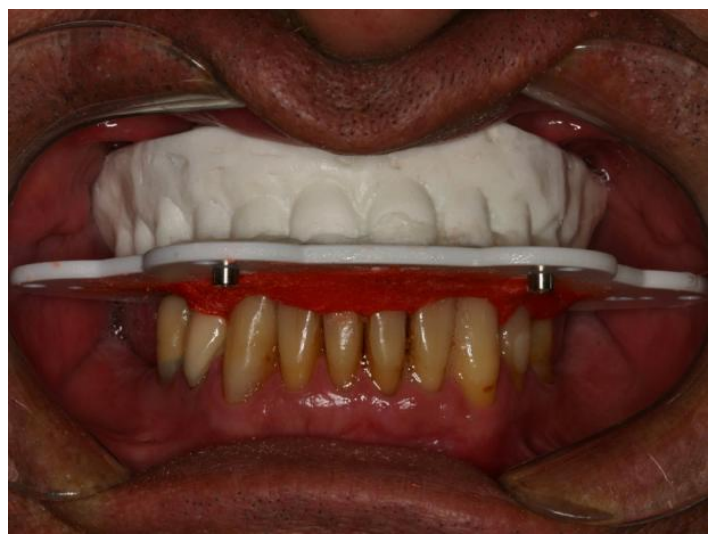

Fig4. Bite registration the tomographic plate

After the examination, the DICOM files originated in the tomography were converted to a new extension, which allows the exploration and execution of the virtual planning in the specific software. Once converted, the threedimensional reconstruction of the patient's maxillofacial skeleton was performed in KEATech software, where the surgery was planned and performed in a virtual environment (Fig. 5). Six implants were planned in the maxilla and after the planning was finalized, the software generated a coordinate report that allows the placement of the guide tubes in the tomographic guide, transforming the tomographic guide into a surgical guide. 


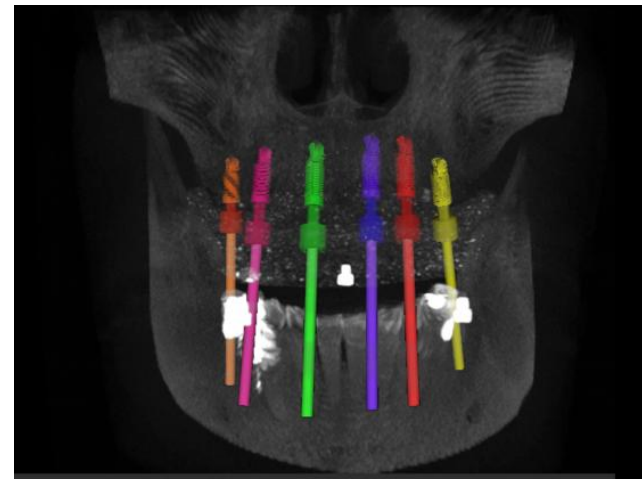

Fig5. Virtual planning on the KEA Tech software

After the placement of all the tubes, a silicone model surgery was performed, where the surgical guide itself was used to prepare the patient's total prosthesis for the surgical procedure (Fig. 6). The guide was placed on the silicone model and the perforations were performed until the long drill of the PROSS GUIDE laboratory kit reached the other side of the cast model (Fig. 7,8), allowing the complete denture being installed on the model and perforated in reverse, preparing the exact region where the implants would be placed later, during the surgical procedure (Fig. 9). After the preparation of the complete denture, the tomo / surgical guide was finished and polished and sterilized in a solution of chlorhexidine at $02 \%$.

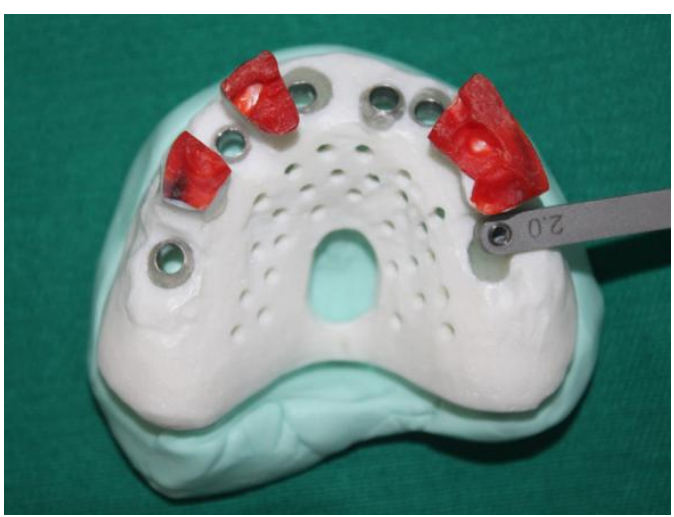

Fig6. Silicone model surgery using the surgical guide

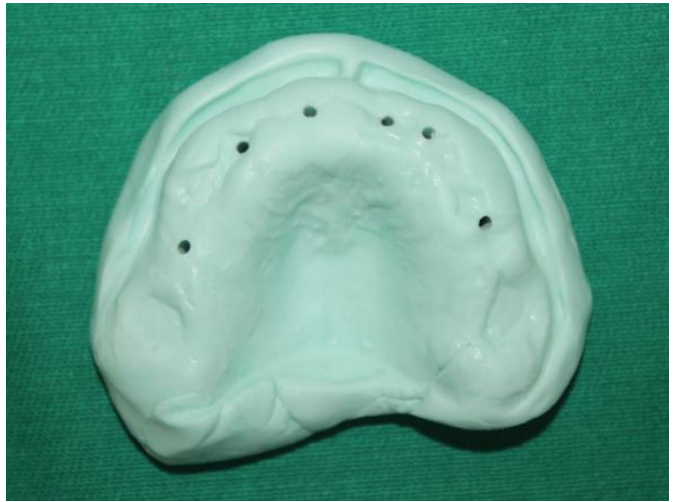

Fig7. Occlusal view of the silicone model after preparation

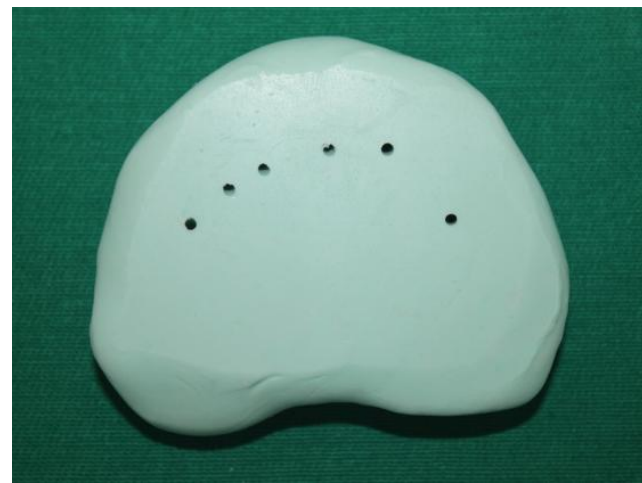

Fig8. Back view of the silicone model after preparation

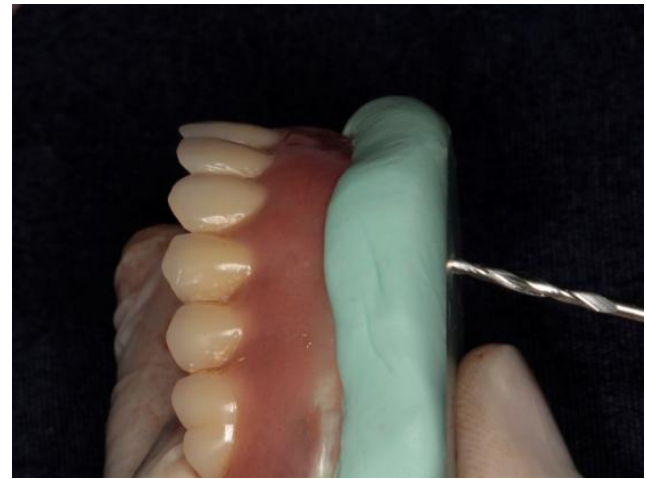

Fig9. Complete denture installed on the model and perforated in reverse

After this process, the surgery is prepared and ready to be started. The patient was anesthetized and the tomo / surgical guide was placed in a position of maxillo-mandibular registration (the same one in which the tomographic examination was performed), the guide was fixed and stabilized using mini-implants with conical mini-abutment connection (PROSS DabiAtlante - RibeirãoPreto, SP, Brazil) (Fig. 10). This procedure allows the guide to be removed and return to its original position as many times as necessary during surgery.

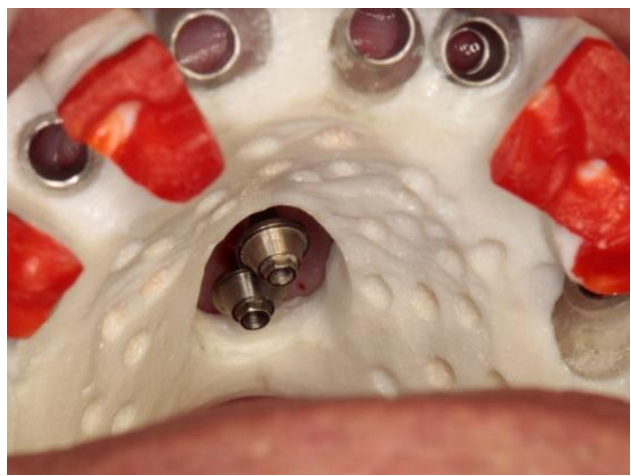

Fig10. Mini-implants with conical mini-abutment connection for fixation of the surgical guide

Thus, with the stabilized guide (Fig. 11), the sequence of perforations was started using the PROSS GUIDE / KEA Tech - DabiAtlante surgical kit, which has colored adjustable stop 
drills coupled to all drills. In this way, it is possible to adjust the size of the drill according to the report obtained through the software (Fig. 12). Each drill has colored labels in its stop drills corresponding to the color of the drill guide according to its diameter. In this way, the identification by the surgeon becomes easier. After surgical instrumentation, sixcylindrical Morse Tapered cylindrical Implants - (PROSS DabiAtlante - RibeirãoPreto, SP, Brazil) were placed. All implants reached primary stability higher than $45 \mathrm{Ncm}$, allowing the application of the concept of immediate loading.

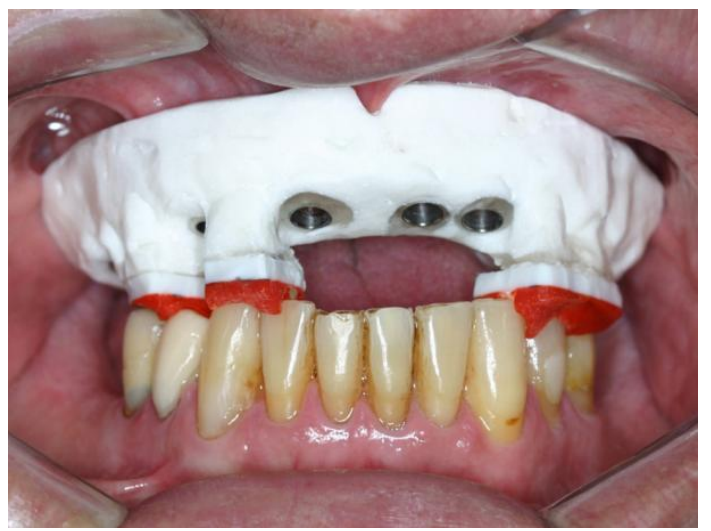

Fig11. The guide fixed and stabilized in bite registration

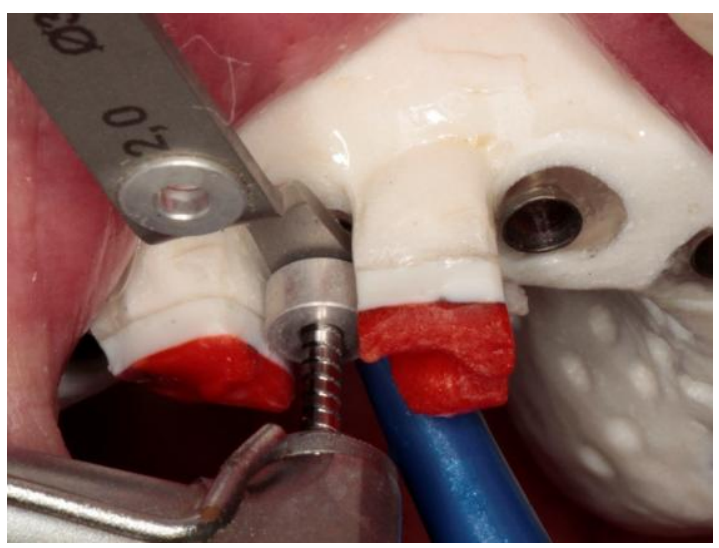

Fig12. Guided perforation with the stabilized guide

SixConical Mini abutments (PROSS DabiAtlante - RibeirãoPreto, SP, Brazil) were then seated with their heights preselected in the software (Fig. 13). After the recommended torque by the manufacturer, the previously prepared complete denture (Fig. 14) was captured using titanium copings (PROSS DabiAtlante - RibeirãoPreto, SP, Brazil). After capturing the position of all copings, with the patient in occlusion (Fig. 15), the complete denture was removed and transformed into a fixed prosthesis (Fig. 16). After transformation, finishing and polishing, the fixed prosthesis was installed and the occlusion was once again checked and adjusted (Fig. 17).

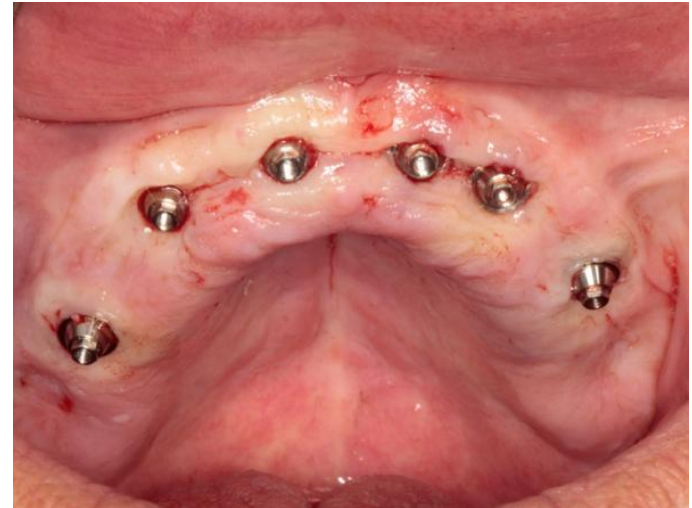

Fig13. Abutments connected to the implants

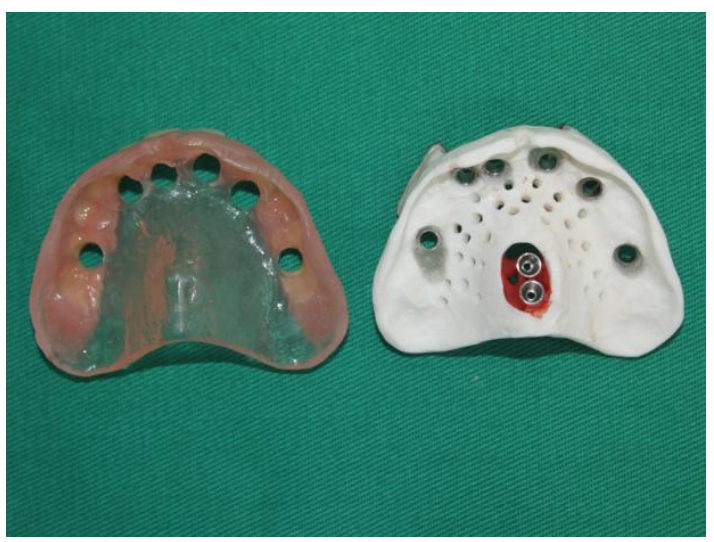

Fig14. Preparation of the complete denture, respecting the surgical guide perforations

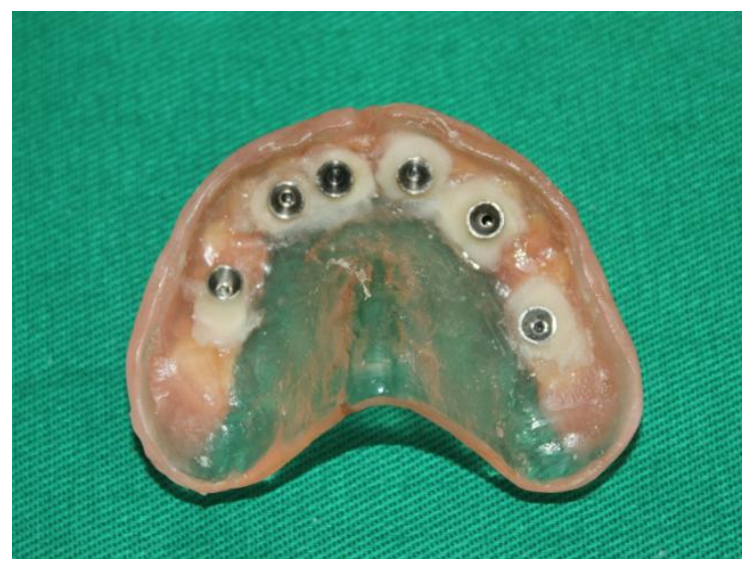

Fig15. After capturing the position of all titanium copings

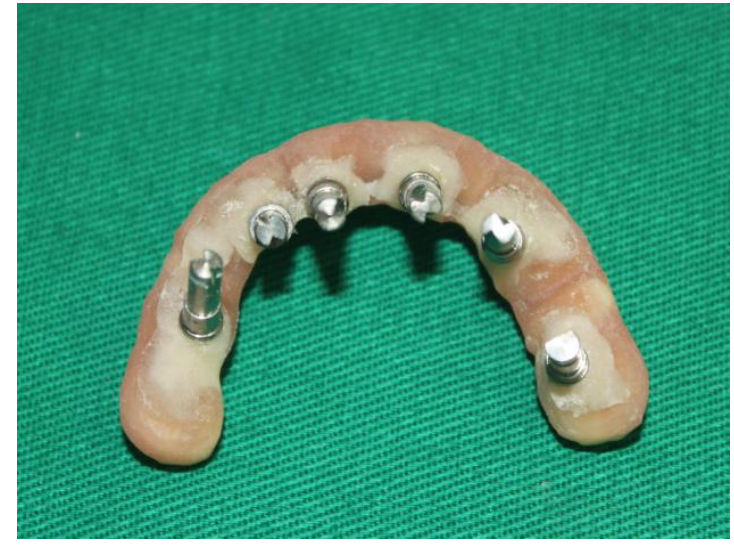

Fig16. Transformation of the complete denture 


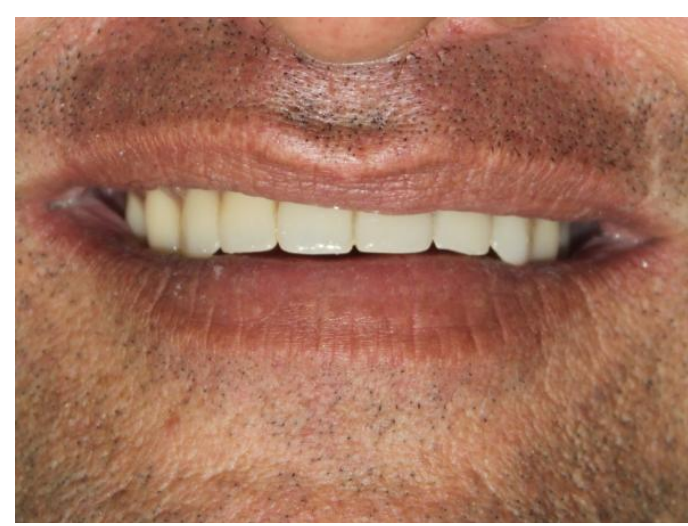

Fig17. Final Result

\section{DISCUSSION}

Many advantages over conventional techniques have been reported by several authors, showing that performing guided surgery, without incision and flap opening, provide better postoperative results when compared to conventional surgery $(7,8)$. In the present case, the patient presented a comfortable postoperative result without the need for analgesics.

Before surgery, it is important to observe the opening of the patient's mouth, since it takes between 4 and $5 \mathrm{~cm}$ to compensate for the thickness of the surgical guide and the length of the drills (8). It is also very important to observe the amount of keratinized mucosa as well as the soft tissue resilience where the implants will be placed. The success and longevity of osseointegrated implants is closely related to the quality of peri-implant tissues. For esthetic and functional resolutions with predictable results, total rehabilitations need a well-executed reverse planning, the prosthesis must be previously designed, respecting a protocol of orofacial esthetic analysis and information obtained from the patient, such as smile line, symmetry and facial proportions, Color, position and dental proportions, vertical dimension of the face, among others. Based on this, it was suggested the option of fixed prosthesis supported by implants with discrete vertical compensation of the protocol type (9), on six implants placed by guided surgery.

During the surgical procedure, the guide should not have any type of movement or scale. It is necessary to maintain the perfect fit and ensure its stability, avoiding the possibility of changing position during surgical instrumentation, one of the main causes of failure during this type of procedure. Much is discussed about the movement during the surgical procedure, however, few authors discuss the importance of precision during the tomographic examination.
It has been previously described that the movement during the tomographic examination can be as important as the movement during the surgical procedure, since all the planning will be based on the images obtained in this examination(9). In systems based on stereolithography, the patient must undergo a tomographic examination and soon afterwards, his tomographic guide must also be scanned by the CT scan. There is also the possibility of doing a tomography of the impression or obtaining of virtual models from the scanning of the cast model, by a laboratory scanner or even by intraoral scanners.

After this procedure, the two images are converted and overlaid into the planning software. Doing two CT scans and an overlap implies the possibility of minor errors and variations that may be vital at the end of treatment. In the PROSS Guide / KEA Tech system -which already has two patents granted in the United States and Australia, this possibility of error is diminished, as the tomographic guide will later be converted into the surgical guide. Thus, only one tomography with the patient in occlusion with the use of a rigid maxillary / mandibular registration is required. In this way, we call the guide astomo / surgical guide, since it is used in both moments. The bite registration is responsible for stability during the tomographic examination and also for the stabilization of the surgical guide during the operative procedure. In this way, it is possible to achieve reproducibility of this position, since the occlusion is physiological and individual for each patient.

Another important point in the application of the surgical technique is the possibility of not only performing guided surgical instrumentation but also the installation of the implants, guided by tubes with different diameters, which increases the installation precision. Depending on the difference in bone density of the area, the implant may suffer a small displacement of its planned trajectory due to the fact that the lower density bone yields more the deformation caused by the installation of the implant, thus changing its initially determined course. For this reason, having final implant prostheses before the surgical procedure is still far from being a reality.

Guided surgery has an important contribution to the prosthetic resolution, reducing the compensation of the original teeth lost due to the implant position closest to the ideal, with a 
preview of the prosthesis in relation to the available bone even before implant placement. This improves the distributions of biomechanical forces, aesthetics, phonetics and the hygienic condition by the patient. A recent meta-analysis of the in vitro and in vivo studies revealed a total mean error of $1.12 \mathrm{~mm}$ at the entry point and $1.39 \mathrm{~mm}$ at the apex (10) and when we are dealing with prosthetic seating accuracy, these mismatches are inadmissible. This protocol allows simplification of the procedure for clinical staff and for the patient. Because it is a minimally invasive procedure, the technique consumes less time and offers less postoperative morbidity, less bleeding and a high degree of patient satisfaction $(8,11,12$, 13). Clinical studies have shown that the success rate of implants in guided surgery is very similar to the conventional technique, with a high absolute survival rate around $95 \%$ (12). However, the rate of implant survival is not synonymous of success. For many times, the implant may be osseointegrated, but in an inadequate position, which may even render its use unfeasible by compromising the prosthesis. A recent study concluded that the inexperience of the performing surgeon did not influence the accuracy of implant placement in edentulous jaws, when experienced professionals supervise all necessary steps for the procedure (14). As previously mentioned, the accuracy of this concept depends on several factors, from the acquisition of the data set to the surgical procedure. In this way, the advantages of having surgery guided by a virtual plan are clear. The Fig. 18 - shows the final radiography, after the implant placement.

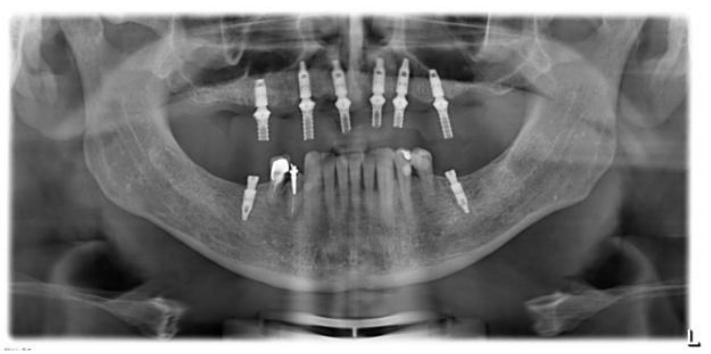

Fig18. Final Radiography

Although it seems extremely simple to perform, it requires technical expertise from the involved staff and detailed planning to avoid any complications during the procedure. Guided surgery can be considered as a safe and predictable alternative for the rehabilitation of fully edentulous jaws when performed within the correct indications and respecting each of the steps until the execution of the surgery and the preparation of the prosthesis. The Figure 19 shows the complete workflow with the chronogram of the total tasks of the procedure. The highlight of this technique is the obligation in detailed planning done prior to the surgery, increasing the quality of the final rehabilitations and reducing the number of intraoperative complications in both surgery and prosthetic resolution.

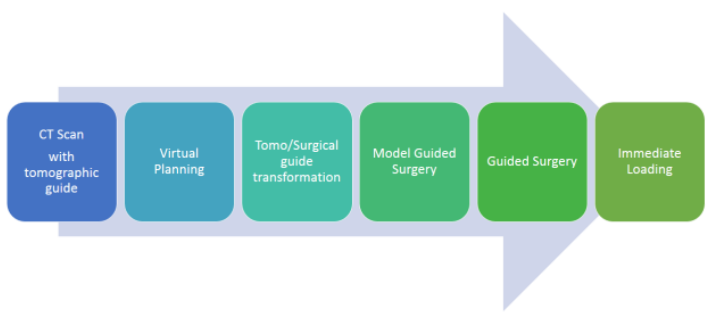

Fig19. Complete workflow - chronogram of the total tasks of the procedure

\section{CONCLuSion}

Expected results were achieved and patient expectations were met. The positioning of the implants showed precision and fidelity to the previous virtual planning in the computer. Virtual planning made possible not only quicker and less invasive surgery, but also facilitated and greatly accelerated the process of making the prosthesis in immediate load, bringing benefits to the clinical staff and the patient undergoing surgery.

\section{REFERENCES}

[1] Patel N. Integrating three-dimensional digital technologies for comprehensive implant dentistry. J AmDent Assoc. 2010; 141: 20-24.

[2] Ganz SD. Presurgical planning with CTderived fabrication of surgical guides. J Oral Maxillofac Surg. 2005; 63: 59-71.

[3] Carneiro TAPN, Oliveira MTF, SimamotoJúnior P, de Paulo LFB, Neves FD and ZanettaBarbosa D.Guided Surgery in a Full Arch Rehabilitation of an Edentulous Maxilla. J Dent \& Oral Disord. 2016; 2(5): 1028.

[4] Sicilia A, Botticelli D; Working Group 3. Computer-guided implant therapy and soft- and hard-tissue aspects. The Third EAO Consensus Conference 2012. Clin Oral Implants Res. 2012 Oct; 23 Suppl 6:157-61.

[5] Pettersson A, Kero T, Gillot L, Cannas B, Fäldt $J$, Söderberg R, Näsström K.Accuracy of CAD/CAM-guided surgical template implant surgery on human cadavers: Part I. J Prosthet Dent. 2010 Jun;103(6):334-42.

[6] Pettersson A, Komiyama A, Hultin M, Näsström K, Klinge B. Accuracy ofvirtually planned and template guided implant surgery 
on edentate patients. Clin Implant Dent Relat Res. 2012 Aug; 14(4):527-37.

[7] Sarment DP, Sukovic P, Clinthorne W. Accuracy of implant placement with a stereolithographic surgical guide. Int $\mathrm{J}$ Oral Maxillofac Implants. 2003; 18: 571-577.

[8] vanSteenberghe D, Glauser R, Blomback U, Andersson M, Schhtyser F, Pettersson A, et al. A computed tomographic scan-derived customized surgical template and fixed prosthesis for flapless surgery and immediate loading of implants in fully edentulous maxillae: a prospective multicenter study. ClinImplantDentRelat Res. 2005; 1: 111-120.

[9] Neves FD, Mendonça G, Fernandes Neto AJ. Analysis of influence of lip line and lip support in esthetics and selection of maxillary implantsupported prosthesis design. J ProsthetDent. 2004; 91: 286-288.

[10] Tahmaseb A, Wismeijer D, Coucke W, Derksen W. Computer technology applicationsin surgical implant dentistry: a systematic review. Int $\mathrm{J}$ Oral Maxillofac Implants. 2014; 29: 25-42.
[11] Sanna AM, Molly L, Van Steenberg D. Immediately loaded CAD/CAM manufactured fixed complete dentures using flapless implant placement procedures a cohort study of consecutive patients. J Prosthet Dent. 2007; 97: 331-339.

[12] Malo P, de AraujoNobre M, Lopes A. The use of computer-guided flapless implant surgery and four implants placed in immediate function to support a fixed denture: preliminary results after a mean follow-up period of thirteen months. J ProsthetDent. 2007; 97: 26-34.

[13] Pozzi A; Tallarico M; Marchetti M; Scarfo B; Esposito M. Computer-guided versus free-hand placement of immediately loaded dental implants: 1-year post-loading results of a multicentre randomized controlled trial. Eur J Oral Implantol 2014;7(3):229-242

[14] Van de Wiele G, Teughels W, Vercruyssen M, Coucke W, Temmerman A, Quirynen M. The accuracy of guided surgery via mucosasupported stereolithographic surgical templates in the hands of surgeons with little experience. Clin Oral Implants Res. 2014.

Citation: Thiago de Almeida Prado Naves Carneiro DDS, MSc \& PhD, Keuler Ferreira Rangel DDS, Eder Ferreira Rangel DDS, Asbel Rodrigues Machado DDS, MSc. Computer-Guided Implant Surgery, an Overview from the Diagnosis to the Rehabilitation. ARC Journal of Dental science. 2017; 2(2): 18-24. doi:dx.doi.org/ 10.20431/2456-0030.0202005.

Copyright: (C) 2017 Authors. This is an open-access article distributed under the terms of the Creative Commons Attribution License, which permits unrestricted use, distribution, and reproduction in any medium, provided the original author and source are credited. 